\title{
Molecular biology in pancreatic ductal adenocarcinoma: implications for future diagnostics and therapy
}

\author{
Kjetil Søreide · Florian Primavesi · Knut J. Labori · Martin M. Watson · Stefan Stättner (D)
}

Received: 21 December 2018 / Accepted: 11 February 2019 / Published online: 5 March 2019

(C) The Author(s) 2019

\begin{abstract}
Summary
Background Novel technology has enabled researchers to better characterize pancreatic cancers at the molecular level. We wanted to explore some of the emerging discoveries, such as molecular subclassification, use of liquid biopsy and use of organoids in cancer assessment.

Methods A literature review with a search specific to the topic, with recent reviews in major journals and a focus on the last 5 years (until December 2018), was done.

Results Pancreatic ductal adenocarcinoma (PDAC) may now be classified into clinical subgroups based on the predominant genomic profiles, but consensus on one classification system is lacking. Several subtypes have been suggested, including categories such as basal-like, stroma-activated, desmoplastic, pure classical and immune classical types. Further refinement may translate into clinically meaningful groups for therapeutic or prognostic purposes. Liquid
\end{abstract}

\section{K. Søreide, MD, PhD}

Department of Gastrointestinal Surgery, Stavanger

University Hospital, Stavanger, Norway

K. Søreide, MD, PhD $\cdot$ M. M. Watson, MSc

Department of Clinical Medicine, University of Bergen,

Bergen, Norway

Gastrointestinal Translational Research Group, Molecular Lab, Stavanger University Hospital, Stavanger, Norway

F. Primavesi, MD · PD Dr. S. Stättner, MD (ه)

Department of Visceral, Transplantation and

Thoracic Surgery, Medical University of Innsbruck,

Anichstraße 35, 6020 Innsbruck, Austria

stefan.staettner@i-med.ac.at

K. J. Labori, MD, PhD

Department of HPB Surgery, Oslo University Hospital,

Rikshospitalet, Oslo, Norway biopsies (by means of circulating cancer cells, cellfree DNA, exosomes or other constituents of cancer cells in blood) may aid in earlier diagnosis, define prognostic groups and even predict therapy response and resistance. Organoids are increasingly used for the opportunity to investigate druggable and effective targets ex vivo and should facilitate personalized and precise, targeted therapy in the near future. While immunotherapy has not yet proved to be effective, a better understanding of molecular subgroups and specific immune profiles may help identify candidates for this approach in a more selective approach.

Conclusion Novel molecular techniques have the potential to accelerate the road to improved outcomes in patients with pancreatic cancer.

Keywords Pancreas - Cancer - Molecular pathology · Genomics · Classification · Liquid biopsy · Organoids · Biomarkers · Druggable targets · Chemotherapy

\section{Main novel aspects}

Pancreatic cancer is increasingly understood at the molecular level, with attempts at subtyping groups with distinct outcomes and potential for therapeutic targets.

Liquid biopsies are investigated for their ability to monitor circulating biomarkers and thus potentially detect pancreatic ductal adenocarcinoma (PDAC) at an earlier stage when disease is resectable with the intent for cure, or to monitor the effect of surgery or chemotherapy over time.

Previous clinical trials for targeted therapy in PDAC have so far failed to show substantial survival benefits. Organoids are increasingly explored for the ability to test multiple drugs in vivo, in order to better target the druggable points in the individual patient's cancer. 
Fig. 1 Risk groups and risk factors for developing PDAC. Risk populations are currently restricted to hereditary genetic syndromes and pancreatic cysts. The risk of pancreatic cancer increases with age, but otherwise the known risk factors are nonspecific (and there is an unmet need for better risk features). An overall goal is to increase resectability by earlier diagnosis and thus improve prognosis and survival. This goal can be facilitated by identification of novel highrisk groups that would be suitable targets for tailored surveillance. (Reprinted from K. Søreide [16], Copyright (2018), with permission from Elsevier)

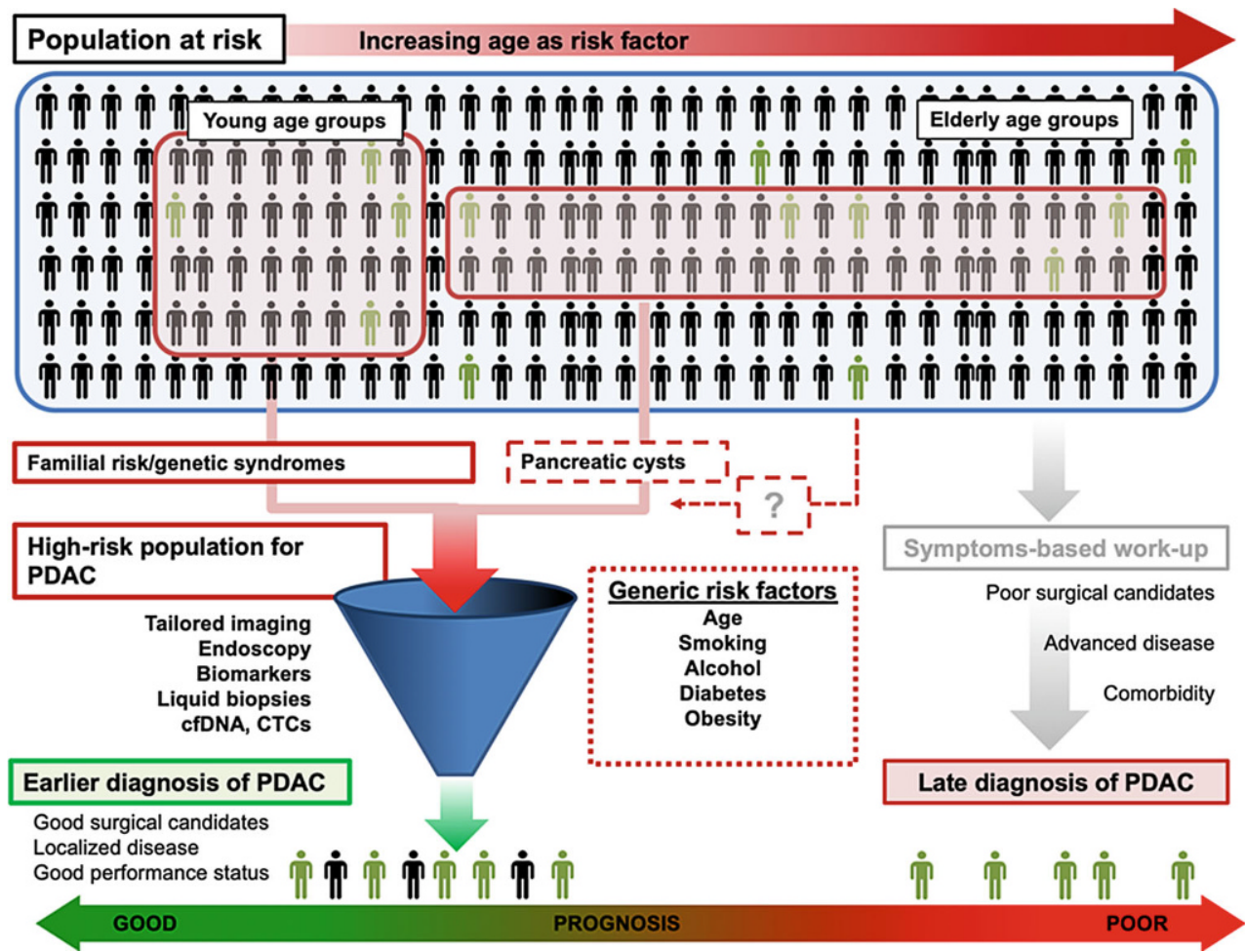

\section{Introduction}

Pancreatic ductal adenocarcinoma (PDAC) remains one of the most lethal of all cancers with mortality almost equal to the incidence of the disease [1]. PDAC is refractory to almost all current therapies. Despite being a devastating disease, only few major changes to its clinical management have been made over the past 50 years to improve prognosis, which remains extremely poor at present [2]. Indeed, due to progress made in other cancer fields combined with an aging population, the predictions are that PDAC will become one of the leading causes of cancer-related deaths in the next decade [3].

One major factor contributing to the dismal prognosis is the late detection in the majority who present with the disease: more than half of patients present with distant metastases at the time of diagnosis; a further one third may have locally advanced or, at best, only borderline resectable disease-hence, usually less than $20 \%$ are amenable to surgical therapy, which is the only real chance of long-term cure. Data on the actual resection rates in population-based registries suggest there is huge variation in clinical practice [4]. PDAC is notoriously resistant to most conventional chemotherapies, although some novel combinations have proven promising, such as FOLFIRINOX in the metastatic setting [5], for borderline disease [6], becoming the preferred standard in the adjuvant setting [7] and, furthermore, now investigated as an neoadjuvant approach to surgery in upfront resectable disease $[8,9]$. Early diagnosis is hampered by the hard-to- access gland positioned in the posterior part of the abdomen. While novel technologies such as endoscopic ultrasound (EUS), computed tomography (CT) and magnetic resonance imaging (MRI) have made visualization of the gland more accurate [10], the criteria for selection of the population to be screened remains debatable [11]. Pancreatic cancer is hereditary and associated with genetic syndromes in but a very small minority of cases [12-14], and definite risk groups do not exist except for some cystic lesions [15] that are known to be premalignant or have malignant potential (Fig. 1). For the rest of the population, the risk factors are generic-smoking, obesity and aging are strongly related to risk of PDAC-and too general to allow for screening of particular persons at risk [16]. Thus, great interest exists in investigating biological factors and novel technologies that can facilitate an earlier diagnosis or be predictive or prognostic for patients with PDAC. The aim of this narrative review is to give a brief insight into some of the molecular mechanisms and technologies that may improve diagnosis and therapy for PDAC in the near future.

\section{Methods}

This narrative review was based on a search of the PubMed database up to December 20, 2018, with a focus on articles written in the English language and published in the past 5 years on the topic of pancreatic cancer biology and novel methods for diagnosis and therapeutic targets. 


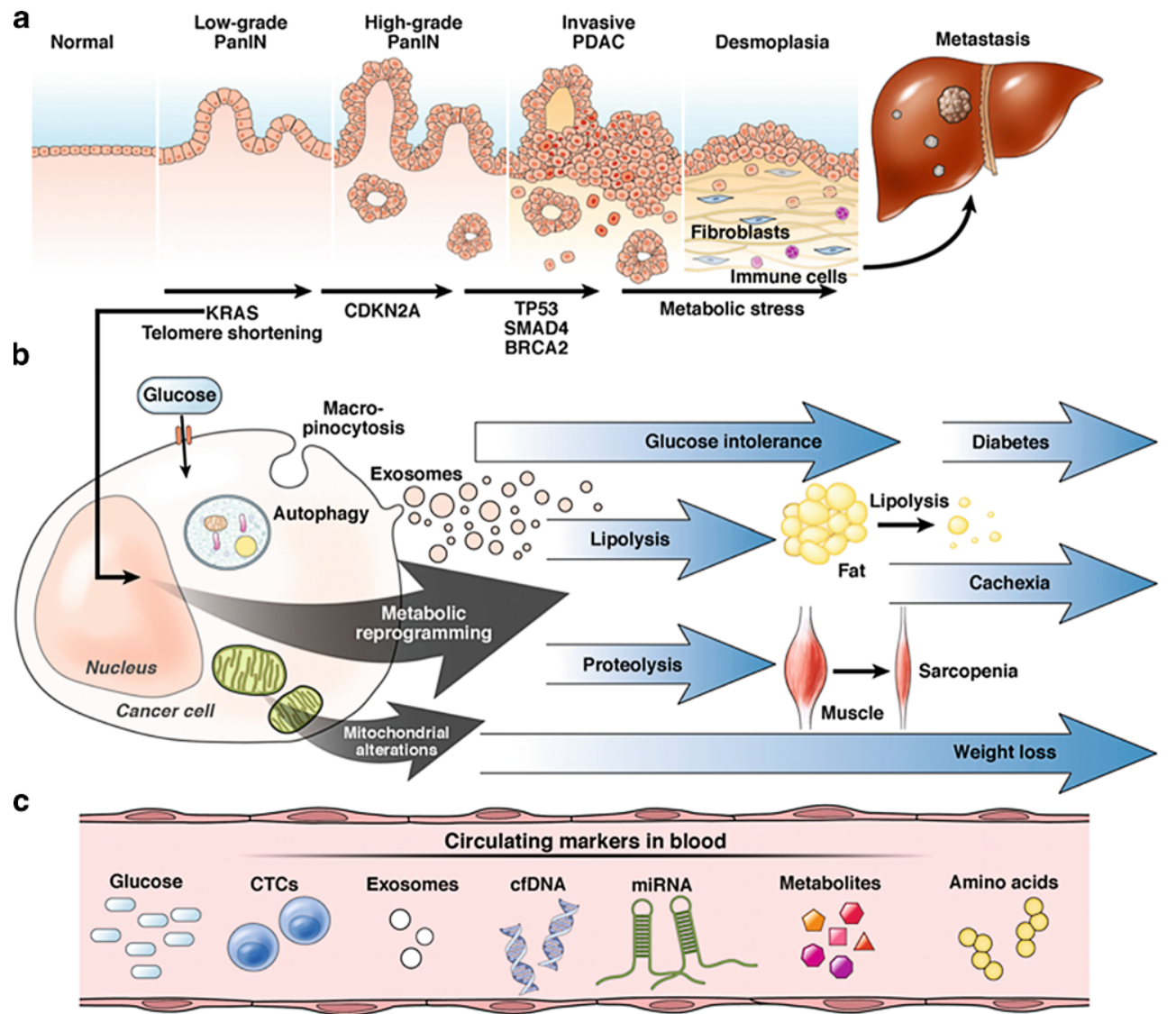

Fig. 2 Pancreatic cancer progression model and opportunity for diagnostic and therapeutic interventions. a Pancreatic cancer progresses through morphologic changes (PanIN) that eventually progress to invasive PDAC. Known genetic alterations occur with each step in the progression. PDAC is further characterized by a strong desmoplastic reaction and an intricate crosstalk between cancer cells and the surrounding fibroblasts and immune cells in the stroma that fosters progression, epithelial-mesenchymal transition and eventually metastasis. b Pancreatic cancer cells are also characterized by a KRAS-driven extensive metabolic reprogramming. This eventually leads to the clinical phenotype of weight loss, diabetes, sarcopenia and cachexia often seen in patients

\section{Increased biological understanding: precursors and genomic classification}

Several steps towards increased understanding of pancreatic cancer have been made over the past decades [14]. Pancreatic cancer most frequently arises from precursor lesions, named pancreatic intraepithelial neoplasia (PanIN) [17]. Pancreatic cancer can also arise from larger precursor lesions such as intraductal papillary mucinous neoplasms and mucinous cystic neoplasms [15]. A stepwise model [18] has been developed to understand the correlation between early morphological changes and associated genetic events [19], as depicted in Fig. 2. With the developments in sequencing technology, the next generation sequencing (NGS) platform has allowed for more widespread with pancreatic cancer. Increases in fasting blood glucose, possibly induced by mechanisms such as adrenomedullinmediated beta-cell destruction, may serve as an early cue to pancreatic cancer. c Assuming that these molecular processes may occur earlier in carcinogenesis and, thus, may potentially be excreted in the circulating blood (c), the identification of such sensitive markers may eventually help to facilitate earlier diagnosis of pancreatic cancer at a curable stage. CTCs circulating tumour cells, cfDNA cell-free DNA, miRNA microRNA, PanIN pancreatic intraepithelial neoplasia, PDAC pancreatic adenocarcinoma. (Reprinted from K. Søreide [16], Copyright (2018), with permission from Elsevier)

and complete characterization of the genomic landscape in PDAC. KRAS is the most consistently affected gene ( $>90 \%$ of tumours) harbouring oncogenic mutations at multiple hotspots, followed closely by CDKN2A, TP53, and SMAD4/DPC4 [20, 21]. The RAS$M A P K$ signalling pathway clearly plays a pivotal role in PDAC development, as up to $60 \%$ of the remaining KRAS wild-type tumours were shown to carry mutations in alternative members such as $B R A F$ and $E R B B 2$ [21].

The improved understanding gained from improved genome and transcriptome analyses has led to the characterization of molecular subtypes [22, 23]. The four subtypes, named "squamous", "pancreatic progenitor", "immunogenic", and "aberrantly differentiated endocrine exocrine" (ADEX) correlate 
with histopathological characteristics and clinical outcomes (e.g. therapeutic response). The squamous tumours are enriched for TP53 and KDM6A mutations, show upregulation of the TP63N transcriptional network, and hypermethylation of pancreatic endodermal cell-fate determining genes. The squamous subclass of PDACs has a poor prognosis [22] and is predominantly associated with cancers of the pancreatic body and tail [24]. Pancreatic progenitor tumours preferentially express genes involved in early pancreatic development (such as FOXA2/3, PDX1 and $M N X 1)$. ADEX tumours displayed upregulation of genes that regulate networks involved in KRAS activation, as well as exocrine (NR5A2 and RBPJL) and endocrine differentiation (NEUROD1 and NKX2-2). Immunogenic tumours contain upregulated immune networks including pathways involved in acquired immune suppression. The four-tier classification scheme has recently been challenged, namely with a somewhat different yet overlapping system of five subtypes suggested, called "pure basal like", "stroma activated", "desmoplastic", "pure classical" and "immune classical" [25]. Subtyping of PDAC into meaningful clinical subgroups is likely to evolve as we learn more about the underlying molecular features and as technical sophistication evolves for further molecular investigation. At the moment, these molecular classifications are not in clinical use, but may aid in better substratification for improved understanding of the underlying biology and clinical behaviour of PDAC.

Also, while the turnaround time and costs of genetic testing have significantly decreased over the past decade, the practical application of molecular results to guide individual patient treatment is currently limited in PDAC. This is in part due to the presence of actionable targets in a relatively small proportion of patients, with studies reporting numbers as low as $26 \%$ of cases [26]. On the other hand, the TGCA consortium has recently reported that up to $42 \%$ of patients carry at least one genetic alteration that could grant inclusion in ongoing clinical trials [21].

\section{A background of bad behaviour in tumour biology}

PDACs are known for their desmoplastic growth pattern, consisting of dense fibrotic reaction, inflammation and poor cellularity. In addition, the poor vessel density within the tumour partly explains both the hypoxic environment (lack of nutrients) and the poor response to most types of chemotherapy. The aggressive nature and early metastatic potential of PDAC sets in with an aggressive perineural growth pattern culminating by early invasion and metastasis, even when cancers are still considered small in size $(<2 \mathrm{~cm})$ [16, 27, 28]. Hence, PDACs have a bad biology even in the very early stages and for rather small tumours [29]. Better and earlier detection is thus crucial for improved outcomes, as well as development of tar- geted drugs that have a higher efficacy to potentially increase the number of resectable cancers.

\section{Potential for earlier detection}

Outside specific genetic syndromes that have a defined risk for PDAC [13], there are no specific risk factors in clinical use for pancreatic cancer. Several techniques and novel diagnostic tools are explored for their ability to detect PDAC at an earlier, resectable and, still, curable stage in the general population. Several promising markers and tools have been proposed over the years, but the actual clinical impact has proven to be slow, with few if any available tests on the market. Many of the tests available take advantage of the deranged metabolism in PDAC, with changes in lipid and protein metabolism as well as development of hyperglycaemia and pre-diabetes in the years prior to detection of PDAC.

Among other tests available are endoscopic sampling and measurements of various biomarkers, ranging from proteins in blood or plasma and genetic mutations sampled in cyst fluid or from pancreatic juice [30] to a proposed "breath test" to measure volatile organic compounds in patients with PDAC [31]. The interest in non-invasive tests stems from the difficult anatomical location of the pancreas, thus the development of a clinically available "liquid biopsy" is particularly of interest (Figs. 2 and 3). This utilises the exploration of circulating markers in blood, for which some promising technologies have recently made this come closer to clinical reality [32-35].

\section{Liquid biopsies}

Any bodily fluid can potentially be used for "liquid biopsy" sampling (e.g. urine, blood, spinal fluid etc), but peripheral blood is the most intensely investigated (Fig. 3a) as it contains circulating tumour cells (CTCs) and circulating tumour-derived cell-free DNA (ctDNA) that may project biological information of a cancer or precancer situation. Methods for enrichment and detection of CTCs and ctDNA, their clinical applications and future opportunities in gastrointestinal cancers are constantly developing, with refinement of technology and detection methods [34, 36]. PDAC is an interesting field for this technology as it may allow for non-invasive testing for an otherwise hardly accessible organ [34, 36-40]. Reports on specific mutations or combinations of markers are promising [35, 37, 39, 41].

Biomarkers that can be detected in peripheral blood and predict the risk of having resectable cancer have become closer to reality with recent studies, such as that of CancerSEEK, showing promising results with clinical applications [32]. Patients with positive CTCs prior to resection of PDAC have a poorer prognosis, even when adjusted for histologically unfavourable factors [42]. 
a

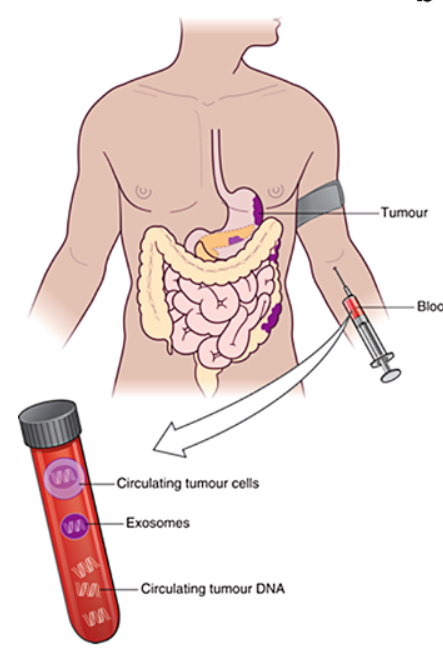

b

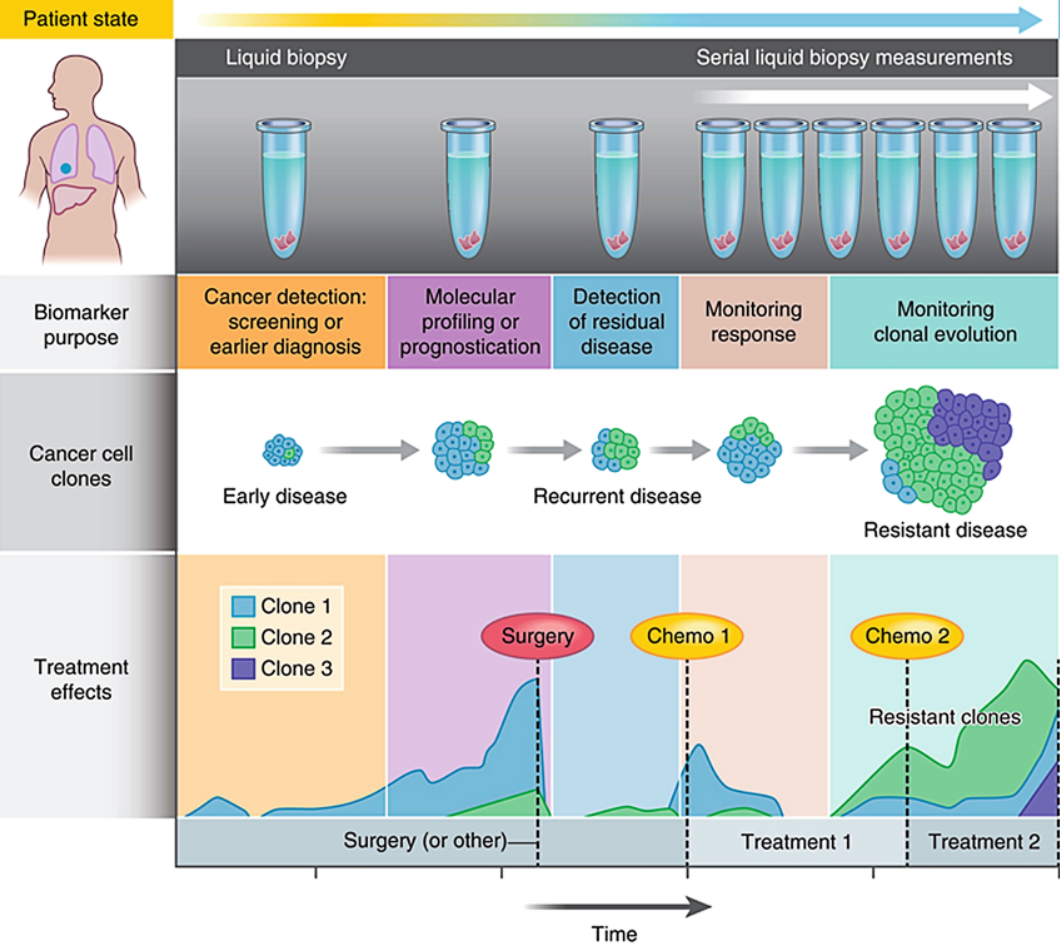

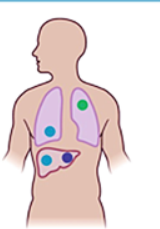

Fig. 3 Liquid biopsy and clinical implications for monitoring and therapeutic intervention. a Liquid biopsies (here represented by blood sample) provide access to the tumour genome by investigating circulating tumour cells circulating cell free DNA, exosomes, microRNA and other genomic and proteomic biomarkers. b Clinical applications of liquid biopsies in cancer management. Examples include diagnostic,

\section{Organoids (patient-derived organoids)}

Traditional monolayer cell lines have proven useful in research in a variety of solid malignancies over the past decades. Nevertheless, these models carry numerous constraints, especially in poorly resectable, low-cellularity cancers such as PDAC, where the issue of representativity, both cellular (intratumoural subpopulations) and at the population level (interpatient tumour heterogeneity) plays a limiting role. Organoid culture methods have been recently established from clinical specimens [43-47] and represent an incredible advantage over monolayer cell lines: they can be generated from a resected PDAC in about 2-4 weeks [43], are amenable to therapeutic screening as well as genetic and biochemical perturbation [48], and are able to recapitulate interactions between tumour and stromal compartment. Because organoids can be generated with high efficiency and speed from fine-needle aspirations, biopsies or resection specimens [43-46], they can serve as a personal cancer model (Fig. 4). The biopsied or resected tumour tissue from the individual patient can be grown and tested for a plethora of potential drugs, only to choose the drug from samples that show any response in the organoid model. Hence, the efficacy may be increased by personalizing the type of therapy given to any patient with PDAC. Per- prognostic or predictive markers. Therapy effect can be monitored and cancer clones followed for response or resistance to therapy, either as a before/after effect of surgery, or as drug-related responses. (Reproduced with permission from Nordgård O et al. [34]. Copyright (2018), with permission from Wiley)

sonalized treatment could soon become a more standard practice by using these cell cultures for extensive molecular diagnosis and drug screening [43, 47, 49]. Drug sensitivity assays can give a clinically actionable sensitivity profile of a patient's cancer. Combined genomic, transcriptomic and therapeutic profiling of patient-derived organoids can identify molecular and functional subtypes of pancreatic cancer, predict therapeutic responses and facilitate precision medicine for patients with PDAC [50].

\section{Molecularly targeted therapies}

Targeting specific mutations in pancreatic cancer has been a matter of research for over several decades now. The difficulties and obstacles that have been encountered during multiple therapeutic approaches to the $R A S / R A F / M E K / E R K$ pathway are exemplarily on the so far fruitless way to an effective, personalized treatment [51]. Although more than $90 \%$ of PDACs harbour a KRAS mutation, inhibition of RAS activation or its' downstream signalling with farnesyltransferase inhibitors (tipifarnib), MEK 1/2 inhibitors (selumetinib, trametinib) alone or in combination with EGFR inhibitors (erlotinib) either did not show a significant benefit in survival or were not clinically applicant due to overlapping toxicities of small molecule inhibitors 


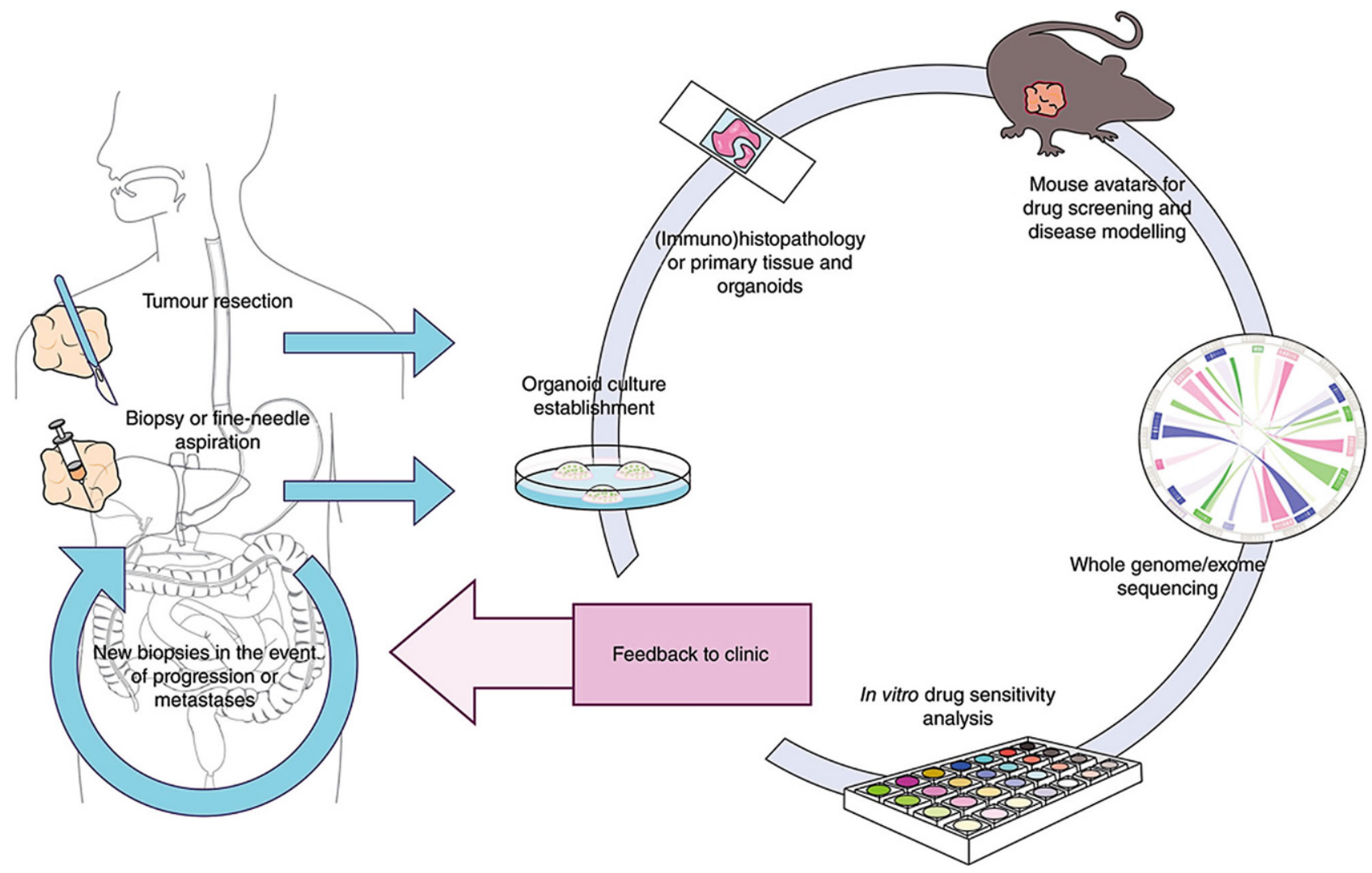

Fig. 4 Model for personalized cancer therapeutics using organoids. Information from gene sequencing, in vitro drug analysis and mouse studies can be combined with traditional molecular analyses, such as immunohistochemistry, to establish a complete tumour profile. As the establishment and complete analysis of an organoid line takes only a few weeks, the

and necessary dose reductions. It has been shown that PDAC possess multiple resistance mechanisms to overcome selective signalling blockades induced by these drugs. Recently, a new strategy to control the "undruggable" KRAS oncogenic pathway has been postulated by inhibition of SIAH (seven in absentia homolog), the most downstream gatekeeper in this deleterious signalling cascade [52]. However, pharmaceutical evaluation of this potential target is still pending to date.

According to a recent systematic review [53], an average yearly number of more than 60 clinical trials have been reported in the last years examining the efficacy of molecularly targeted therapies in unresectable PDAC, most commonly studying inhibitors of EGFR, VEGF, RAS pathways and tyrosine kinases. Especially EGFR inhibitors such as erlotinib, cetuximab and panitumumab have been extensively studied with phase I/II trials in this setting, albeit with limited numbers of patients and without a clear breakthrough in terms of overall or progression-free survival. Further results of phase III studies are therefore eagerly awaited. information could be returned swiftly to the clinic. When patients develop metastases or the disease relapses, new tumour biopsies can be taken and new organoid lines established. (Reproduced with permission from Aberle MR et al. [48]. Copyright (2018), with permission from Wiley)

\section{Immunotherapy}

As immune evasion is a well-described hallmark of cancer development, interest in targeting mechanisms that can trigger the immune system to attack cancer cells has gained increasing interest. Notably, immunotherapy has proved effective for some cancers, and changed the standard of care and life expectancy for several malignancies, such as cancers of the lung, head and neck, gastrointestinal tract, and for some colorectal cancers. Disappointingly, singleagent immunotherapy has had little effect in PDAC [54]. Increasing evidence suggests that the PDAC microenvironment is comprised of an intricate network of signals between immune cells, PDAC cells and stroma, resulting in an immunosuppressive environment resistant to single-agent immunotherapies [54, 55]. It has also been shown that a higher density of CD3+ T-cells in the stroma is associated with longer progression-free survival in patients with PDAC [56], and that combination of mutational profiles and immune markers may define subgroups of patients who may respond to specific types of therapies [57]. These findings are promising and may represent potential 
paradigm changes in the approach to how individual patients with PDAC may be treated in the future.

\section{Conclusion}

Pancreatic adenocarcinoma (PDAC) is a lethal malignancy that is refractory to all current therapies. Research into the mechanisms driving this cancer is the key to developing better diagnostic and treatment options. PDAC may now be classified into clinical subgroups based on the predominant genomic profiles, but consensus on one classification system is still lacking. Both four and five subtypes have been suggested, including basal like, stroma activated, desmoplastic, pure classical and immune classical types. Further refinement may translate into clinical meaningful groups for therapeutic or prognostic purposes. Liquid biopsies (by means of circulating cancer cells, cell-free DNA, exosomes or other constituents of cancer cells in blood) may aid in earlier diagnosis, define prognostic groups and even predict therapy response and resistance. Organoids are increasingly evaluated for their ability to investigate druggable and effective targets in any individual patient and should facilitate personalized and precise, targeted therapy in the near future. Since most molecularly targeted therapies have not shown substantial benefit in clinical trials so far, some of the new molecular techniques raise hope of having the potential to accelerate improved outcomes in patients with pancreatic cancer.

Acknowledgements Open access funding provided by University of Innsbruck and Medical University of Innsbruck

Funding Open access funding provided by University of Innsbruck and Medical University of Innsbruck.

Conflict of interest K. Søreide, F. Primavesi, K.J. Labori, M.M. Watson and S. Stättner declare that they have no competing interests.

Open Access This article is distributed under the terms of the Creative Commons Attribution 4.0 International License (http://creativecommons.org/licenses/by/4.0/), which permits unrestricted use, distribution, and reproduction in any medium, provided you give appropriate credit to the original author(s) and the source, provide a link to the Creative Commons license, and indicate if changes were made.

Publisher's Note Springer Nature remains neutral with regard to jurisdictional claims in published maps and institutional affiliations.

\section{References}

1. Kamisawa T, Wood LD, Itoi T, et al. Pancreatic cancer. Lancet. 2016;388:73-85.

2. Søreide K, Aagnes B, Moller B, et al. Epidemiology of pancreatic cancer in Norway: Trends in incidence, basis of diagnosis and survival 1965-2007. Scand J Gastroenterol. 2010;45:82-92.

3. Rahib L, Smith BD, Aizenberg R, et al. Projecting cancer incidence and deaths to 2030: The unexpected burden of thyroid, liver, and pancreas cancers in the United States. Cancer Res. 2014;74:2913-21.

4. Huang L, Jansen L, BalavarcaY, etal. Resection of pancreatic cancer in Europe and USA: An international large-scale study highlighting large variations. Gut. 2019;68:130-9.

5. Conroy T, Desseigne F, Ychou M, et al. FOLFIRINOX versus gemcitabine for metastatic pancreatic cancer. N Engl J Med. 2011;364(19):1817-25. https://doi.org/10.1056/ nejmoal011923.

6. MurphyJE, Wo JY, Ryan DP, et al. Total Neoadjuvant therapy with FOLFIRINOXfollowed by individualized Chemoradiotherapy for borderline Resectable pancreatic Adenocarcinoma: A phase 2 clinical trial. Jama Oncol. 2018;4:963-9.

7. Conroy T, Hammel P, Hebbar M, et al. FOLFIRINOX or Gemcitabine as Adjuvant therapy for pancreatic cancer. N Engl J Med. 2018;379(25):2395-406. https://doi.org/10. 1056/nejmoa1809775.

8. Del Chiaro M, Soreide K. Trials and tribulations of neoadjuvant therapy in pancreatic cancer. $\mathrm{Br} J$ Surg. 2018;105:1387-9.

9. Versteijne E, Vogel JA, Besselink MG, et al. Meta-analysis comparing upfront surgery with neoadjuvant treatment in patients with resectable or borderline resectable pancreatic cancer. Br J Surg. 2018;105:946-58.

10. Paulson AS, Tran Cao HS, Tempero MA, et al. Therapeutic advances in pancreatic cancer. Gastroenterology. 2013;144(6):1316-26. https://doi.org/10.1053/j. gastro.2013.01.078.

11. Paiella S, Capurso G, Cavestro GM, et al. Results of first-round of surveillance in individuals at high-risk of pancreatic cancer from the AISP (Italian association for the study of the pancreas) registry. Am J Gastroenterol. 2018; https://doi.org/10.1038/s41395-018-0414-z.

12. Shin EJ, Canto MI. Pancreatic cancer screening. Gastroenterol Clin North Am. 2012;41:143-57.

13. Whitcomb DC, Shelton CA, Brand RE. Genetics and genetic testing in pancreatic cancer. Gastroenterology. 2015;149(5):1252-64. https://doi.org/10.1053/j.gastro. 2015.07.057.

14. Ryan DP,Hong TS, Bardeesy N.Pancreatic adenocarcinoma. NEngl J Med. 2014;371:1039-49.

15. Tanaka M. Intraductal papillary Mucinous neoplasm as the focus for early detection of pancreatic cancer. Gastroenterology. 2018;154(3):475-8. https://doi.org/10.1053/j. gastro.2018.01.014.

16. Søreide K. Sweet predictions speak volumes for early detection of pancreatic cancer. Gastroenterology. 2018;155(2):265-8. https://doi.org/10.1053/j.gastro.2018. 06.054 .

17. Hruban RH, Adsay NV, Albores-Saavedra J, et al. Pancreatic intraepithelial neoplasia: a new nomenclature and classification system for pancreatic duct lesions. Am J Surg Pathol. 2001;25:579-86.

18. Hruban RH, Goggins M, Parsons J, et al. Progression model for pancreatic cancer. Clin Cancer Res. 2000;6:2969-72.

19. Klein WM, Hruban RH, Klein-Szanto AJ, et al. Direct correlation between proliferative activity and dysplasia in pancreatic intraepithelial neoplasia (PanIN): Additional evidence for a recently proposed model of progression. Mod Pathol. 2002;15:441-7.

20. Borazanci E, Dang CV, Robey RW, et al. Pancreatic cancer: "A riddle wrapped in a mystery inside an enigma". Clin Cancer Res. 2017;23:1629-37.

21. Raphael BJ, Hruban RH, Aguirre AJ, et al. Integrated genomic characterization of pancreatic ductal Adenocarcinoma. Cancer Cell. 2017;32:185-203. 
22. Bailey P, Chang DK, Nones K, et al. Genomic analyses identify molecular subtypes of pancreatic cancer. Nature. 2016;531:47-52.

23. Waddell N, Pajic M, Patch AM, et al. Whole genomes redefine the mutational landscape of pancreatic cancer. Nature. 2015;518:495-501.

24. Dreyer SB, Jamieson NB, Upstill-Goddard R, et al. Defining the molecular pathology of pancreatic body and tail adenocarcinoma. Br JSurg. 2018;105:e183-e91.

25. Puleo F, Nicolle R, Blum Y, et al. Stratification of pancreatic ductal Adenocarcinomas based on tumor and Microenvironment features. Gastroenterology. 2018;155(6):1999-2013. https://doi.org/10.1053/j.gastro. 2018.08.033.

26. Lowery MA, Jordan EJ, Basturk O, et al. Real-time genomic profiling of pancreatic ductal Adenocarcinoma: Potential actionability and correlation with clinical phenotype. Clin Cancer Res. 2017;23:6094-100.

27. Ansari D, Bauden M, Bergstrom S, et al. Relationship between tumour size and outcome in pancreatic ductal adenocarcinoma. BrJSurg. 2017;104:600-7.

28. di Magliano MP, Logsdon CD. Roles for KRAS in pancreatic tumor development and progression. Gastroenterology. 2013;144(6):1220-9. https://doi.org/10.1053/j.gastro. 2013.01.071.

29. Makohon-Moore A, Iacobuzio-Donahue CA. Pancreatic cancer biology and genetics from an evolutionary perspective. NatRev Cancer. 2016;16:553-65.

30. Springer S, Wang Y, Dal Molin M, et al. A combination of molecular markers and clinical features improve the classification of pancreatic cysts. Gastroenterology. 2015;149(6):1501-10. https://doi.org/10.1053/j. gastro.2015.07.041.

31. Markar SR, BrodieB, Chin ST, et al. Profile of exhaled-breath volatile organic compounds to diagnose pancreatic cancer. BrJSurg. 2018;105:1493-500.

32. Cohen JD, Li L, Wang Y, et al. Detection and localization of surgically resectable cancers with a multi-analyte blood test. Science. 2018;359:926-30.

33. Lennon AM, Wolfgang CL, Canto MI, et al. The early detection of pancreatic cancer: What will it take to diagnose and treat curable pancreatic neoplasia? Cancer Res. 2014;74:3381-9.

34. Nordgard O, Tjensvoll K, Gilje B, et al. Circulating tumour cells and DNA as liquid biopsies in gastrointestinal cancer. BrJSurg. 2018;105:e110-e20.

35. Lapin M, Oltedal S, Tjensvoll K, et al. Fragment size and level of cell-free DNA provide prognostic information in patients with advanced pancreatic cancer. J Transl Med. 2018;16:300.

36. Samandari M, Julia MG, Rice A, et al. Liquid biopsies for management of pancreatic cancer. Transl Res. 2018;201:98-127.

37. Yang S, Che SP, Kurywchak P, et al. Detection of mutant KRAS and TP53 DNA in circulating exosomes from healthy individuals and patients with pancreatic cancer. Cancer BiolTher. 2017;18:158-65.

38. Bernard V, Kim DU, San Lucas FA, et al. Circulating nucleic acids are associated with outcomes of patients with pancreatic cancer. Gastroenterology. 2019;156(1):108-18. https://doi.org/10.1053/j.gastro.2018.09.022.

39. Cohen JD, Javed AA, Thoburn C, etal. Combined circulating tumor DNA and protein biomarker-based liquid biopsy for the earlier detection of pancreatic cancers. Proc Natl Acad SciUSA. 2017;114:10202-7.

40. Shen SY, Singhania R, Fehringer G, et al. Sensitive tumour detection and classification using plasma cell-free DNA methylomes. Nature. 2018;563(7732):579-83. https://doi. org/10.1038/s41586-018-0703-0.

41. Effenberger KE, SchroederC, Hanssen A, etal. Improved risk stratification by circulating tumor cell counts in pancreatic cancer. Clin Cancer Res. 2018;24:2844-50.

42. Hugenschmidt H, Labori KJ, Brunborg C, et al. Circulating tumor cells are an independent predictor of shorter survival in patients undergoing resection for pancreatic and Periampullary Adenocarcinoma. Ann Surg. 2018; https://doi. org/10.1097/sla.0000000000003035.

43. Baker LA, Tiriac H, Tuveson DA. Generation and culture of human pancreatic ductal Adenocarcinoma Organoids from Resected tumor specimens. Methods Mol Biol. 2019;1882:97-115.

44. Zhang F, Wang W, Long Y, et al. Characterization of drug responses of mini patient-derived xenografts in mice for predicting cancer patient clinical therapeutic response. Cancer Commun. 2018;38(1):60. https://doi.org/10.1186/ s40880-018-0329-5.

45. Tsai S, McOlash L, Palen K, et al. Development of primary human pancreatic cancer organoids, matched stromal and immune cells and 3D tumor microenvironment models. BMCCancer. 2018;18:335.

46. Tiriac H, Bucobo JC, Tzimas D, et al. Successful creation of pancreatic cancer organoids by means of EUS-guided fineneedle biopsy sampling for personalized cancer treatment. GastrointestEndosc. 2018;87:1474-80.

47. Moreira L, Bakir B, Chatterji P, et al. Pancreas 3D Organoids: Current and future aspects as a research platform for personalized medicine in pancreatic cancer. Cell Mol Gastroenterol Hepatol. 2018;5:289-98.

48. Aberle MR, Burkhart RA, Tiriac H, et al. Patient-derived organoid models help define personalized management of gastrointestinal cancer. BrJ Surg. 2018;105:e48-e60.

49. Romero-Calvo I, Weber CR, Ray M, et al. Human Organoids share structural and genetic features with primary pancreatic Adenocarcinoma tumors. Mol Cancer Res. 2018;17(1):70-83. https://doi.org/10.1158/15417786.mcr-18-0531.

50. Tiriac H, Belleau P, Engle DD, et al. Organoid profiling identifies common responders to chemotherapy in pancreatic cancer. Cancer Discov. 2018;8:1112-29.

51. Amanam I, Chung V. Targeted therapies for pancreatic cancer. Cancers (Basel). 2018;10(2):36. https://doi.org/10. $3390 /$ cancers 10020036.

52. Van Sciver RE, Lee MP, Lee CD, et al. A new strategy to control and eradicate "Undruggable" oncogenic K-RASdriven pancreatic cancer: Molecular insights and core principles learned from developmental and evolutionary biology. Cancers (Basel). 2018;10(5):142. https://doi.org/ $10.3390 /$ cancers 10050142 .

53. Sheahan AV, Biankin AV, Parish CR, et al. Targeted therapies in the management of locally advanced and metastatic pancreatic cancer: A systematic review. Oncotarget. 2018;9:21613-27.

54. Johnson BA 3rd, Yarchoan M, Lee V, et al. Strategies for increasing pancreatic tumor Immunogenicity. Clin Cancer Res. 2017;23:1656-69.

55. Andrikou K, PeterleC, Pipitone S, etal. Emerging antibodies for the treatment of pancreatic cancer. Expert Opin Emerg Drugs. 2017;22:39-51.

56. Mahajan UM, Langhoff E, Goni E, et al. Immune cell and Stromal signature associated with progression-free survival of patients with Resected pancreatic ductal Adenocarcinoma. Gastroenterology. 2018;155(5):1625-39. https:// doi.org/10.1053/j.gastro.2018.08.009. 
57. Wartenberg M, Cibin S, Zlobec I, et al. Integrated genomic and Immunophenotypic classification of pancreatic cancer reveals three distinct subtypes with prognostic/predictive significance. Clin Cancer Res. 2018;24:4444-54. 